\title{
EL DESEMPEÑO DE LA MUJER EN LA INTELIGENCIA ARTIFICIAL Y SU CONTRIBUCIÓN CON LA ROBÓTICA
}

AUTORES: John Denys Reina Paredes ${ }^{1}$

$$
\begin{aligned}
& \text { Gema Julissa Pinargote Vinces }{ }^{2} \\
& \text { Camila Yanlisbeth Pin Menéndez }{ }^{3} \\
& \text { Dayana Lilibeth Pérez Chilán }{ }^{4} \\
& \text { Kirenia Maldonado Zúñiga }^{5}
\end{aligned}
$$

DIRECCIÓN PARA CORRESPONDENCIA: reina-john8673@unesum.edu.ec

Fecha de recepción: 10/01/2021

Fecha de aceptación: 20/06/2021

\section{RESUMEN}

La presente investigación está enfocada en dar a conocer, porque en el mundo de la tecnología no existe un enfoque neutro con el género femenino, en cuanto al desarrolló de la Inteligencia Artificial(IA) en contribución con los robots; para conseguir cogniciones del porque existe este inconveniente, se investiga en las diferentes fuentes bibliográficas; además se indagará estadísticamente el porcentaje promedio sobre las mujeres que laboran en el campo de la IA y la creación de autómatas en determinados países. El objetivo primordial de esta investigación científica es proporcionar información sobre el escaso desempeño de la mujer en la IA y la contribución con la robótica. En cuanto al desarrollo de este trabajo investigativo se manifiestan, las posibles falencias por las cuales se presenta esta anomalía, empleando la revisión bibliográfica como metodología. El análisis de la investigación, demostró que actualmente existe un 3\% de mujeres que realizan tareas específicas en el sector tecnológico.

PALABRAS CLAVE: Artificial; Inteligencia; Tecnológico.

\section{WOMEN'S PERFORMANCE IN ARTIFICIAL INTELLIGENCE AND ITS CONTRIBUTION TO ROBOTICS}

\footnotetext{
${ }^{1}$ Estudiante de la Universidad Estatal del Sur de Manabí, Facultad de Ciencias Técnicas, Jipijapa, Manabí, Ecuador. reina-john8673@unesum.edu.ec ORCID ID: https://orcid.org/0000-0003-0942-4347

${ }^{2}$ Estudiante de la Universidad Estatal del Sur de Manabí, Facultad de Ciencias Técnicas, Jipijapa, Manabí, Ecuador. pinargote-gema7822@unesum.edu.ec ORCID ID: https://orcid.org/0000-0003-1544-4782

${ }^{3}$ Estudiante de la Universidad Estatal del Sur de Manabí, Facultad de Ciencias Técnicas, Jipijapa, Manabí, Ecuador. pin-camila4758@unesum.edu.ec ORCID ID: https://orcid.org/0000-0001-8074-2351

${ }^{4}$ Estudiante de la Universidad Estatal del Sur de Manabí, Facultad de Ciencias Técnicas, Jipijapa, Manabí, Ecuador. perez-dayana0780@unesum.edu.ec ORCID ID: https://orcid.org/0000-0001-8099-7645

${ }^{5}$ Máster en Ciencias de la Educación, Licenciada en Educación Informática. Docente de la carrera en Ingeniería en Tecnologías de la Información. Universidad Estatal del Sur de Manabí. Jipijapa, Manabí, Ecuador. kirenia.maldonado@unesum.edu.ec ORCID ID: https://orcid.org/0000-0002-3764-5633
} 


\begin{abstract}
The present investigation is focused on making known, because in the world of technology there is no neutral approach with the female gender, in terms of the development of Artificial Intelligence (AI) in contribution to robots; to get cognitions of why this problem exists, it is investigated in the different bibliographical sources; In addition, the average percentage of women working in the field of AI and the creation of automatons in certain countries will be statistically investigated. The primary objective of this scientific research is to provide information on the poor performance of women in AI and the contribution with robotics. Regarding the development of this research work, the possible shortcomings by which this anomaly is presented, using the literature review as a methodology, are manifested. The research analysis showed that there are currently $3 \%$ of women who perform specific tasks in the technology sector.
\end{abstract}

KEYWORDS: Artificial; Intelligence; Technological.

\title{
INTRODUCCIÓN
}

En la actualidad, la Inteligencia Artificial (IA) y la robótica, son disciplinas que poseen un enorme potencial para transformar cualquier aspecto de la vida. Por lo general, en el ámbito tecnológico el perfeccionamiento de los robots ha estado presenta desde hace muchos años atrás, sin embargo, la IA y la tecnología de sensores, se han convertido en bases fundamentales para la creación de autómatas completos, pero es notorio que son los hombres quienes están al mando de cada uno de estos proyectos, mientras que, existe una brecha de genero feminista.

Por este motivo, el objetivo primordial de esta investigación consiste en proporcionar información sobre el desempeño de la mujer en el ámbito de la inteligencia computacional en contribución con la robótica, así mismo, se pretende analizar los posibles problemas o estereotipos que nos les permite a las féminas, laborar en áreas tecnológicas, además, se investiga en diferentes fuentes si es necesaria la participación de la mujer en el desarrollo de las inteligencias artificiales.

En cuanto al desarrollo de esta investigación se plantean interrogantes del ¿porque la inteligencia artificial y los sistemas autómatas discriminan a las mujeres?, y que, aunque las tecnologías creadas por hombres son reconocidas como líderes femeninas en tecnología, la ausencia de las mujeres en el desarrollo de la IA es muy notoria, para detallar cada una de estas anomalías, se emplearon los métodos teóricos de la investigación científica tales como: histórico-lógico y análisis-síntesis. Por consiguiente, el análisis de esta investigación determinó que existe un 3\% de féminas en todo el mundo que son capaces de desarrollar programas de Inteligencia Artificial y Autómatas complejos.

El impacto que asumirá está investigación en cuanto al ámbito de la tecnología, es presentar la situación actual que sufren las mujeres al querer formar parte de una organización que les permita desarrollar aplicaciones de inteligencia artificial y robótica, brindando posibles soluciones e iniciativas tecnológicas para que se termine con los desafíos o estereotipos presentes en el mundo actual.

\section{DESARROLLO}

\section{La Inteligencia Artificial y mujeres}

68 UNESUM-Ciencias. Publicación cuatrimestral. Vol. 5, Año 2021, No. 4 (Número Especial) 
El ámbito de la Inteligencia artificial en conjunto con la robótica se ha ido expandiendo significativamente en los diferentes países del mundo, influyendo de manera rápida y directa en la vida de los seres humanos, es decir, lo que antes solo se podía observar en las telillas de ciencia y ficción, ahora se las puede apreciar en masivas aplicaciones o sistemas informáticos. Sin embargo, a pesar del continuo dominio de esta tecnología, las damas forman parte tal del $12 \%$ de peritos en esta área, según la UNESCO y EQUALS, organismos encargados de promover la igual de géneros en áreas tecnológicas. Es necesario recalcar, que la poca participación de las mujeres en la aplicación de las nuevas tecnologías, presentan anomalías en la creación de programas informáticos que están diseñados para establecer una conversación, puesto que, no se les inculca códigos morales y al no ser controlados en tiempo real, pueden causar un sinnúmero de perjuicios en la sociedad.

\section{Discriminación de la mujer en la Inteligencia Artificial}

Recientemente un chatbot desarrollado por Microsoft absorbió muchos datos mediante la aplicación Twitter y en menos de 15 horas de ser lanzado al mercado tuvo que ser eliminado, dado que, se refería a las féminas como un culto o cáncer. Por esta razón, los expertos acentúan que es necesaria la participación de las mujeres en la implantación de los códigos de manera técnica. Por otra parte, los demás asistentes virtuales como Alexa o Google al realizarles preguntas sobre si es bruta o inútil, es una muestra de sesgos de género, puesto que, el hecho que no responda, no quiere decir que no sea notorio la falta de códigos morales en los sistemas inteligentes; además, se ha descubierto que cuando un aparato informático da una orden, generalmente la voz que se proyecta es de carácter masculino, mientras que si se trata de encender la luz, cambiar de canción, los asistentes de voz son femeninos, esto se da gracias a que los hombres se les hace difícil cumplir con las indicaciones dadas por una fémina, por ello es increíble como la desigualdad de género se encuentra presente en cada innovación tecnológica que se lanza al mercado.

\section{¿Por qué la inteligencia artificial y los sistemas autómatas discriminan a las mujeres?}

En 2014 Amazon estrenó un algoritmo para reclutar nuevos trabajadores en sus almacenes. La herramienta puntuaba de una a cinco estrellas a los mejores candidatos. Todo parecía ideal: la inteligencia artificial (IA) ahorraría horas al departamento de recursos humanos. Pero un año más tarde, la multinacional se dio cuenta que, en los puestos técnicos, como el de desarrollador de software, no se había contratado a ninguna mujer. ¿Acaso no había ninguna candidata con aptitudes? Esto es un claro ejemplo de lo errores que ha venido desarrollando la IA, es decir existen varias empresas que para aprender sobre los estereotipos femenino y masculinos, usaron una másica cantidad de información de Google que determino que cuando ellos eran programadores informáticos, ellas eran amas de casa, y tiene su lógica dado que, en los medios periodísticos de EEUU los presentadores son hombres. Por otro lado, las transparencias de los estereotipos tampoco se encuentran presentes en los sistemas de textos que se usan para aprender, como el traductor google que al escribir que en turco que ella es una doctora lo contrapone traduciendo que él es un doctor. (Peiró, 2019)

\section{Los problemas sociales que no les permite a las féminas laborar en áreas tecnológicas}


Algunos de las dificultades sociales incorporadas en la mujer y la tecnología, son el aislamiento social, brecha digital, y la brecha de género digital.

El aislamiento social vinculado al uso de las TICS, representa un importante problema social. Esto debido a que el uso de estos espacio de relación, en ocasiones, contribuye a la individualización, favoreciendo interacciones impersonales y deteriorando las relaciones con otros grupos sociales, como la familia, la comunidad, compañeros/as de trabajo, así mismo, la brecha digital que se centra en la accebilidad de las TICs y la utilización que hace de ellas, y por último, la diferencia entre mujeres y hombres en el acceso a las TICs, que representa la brecha digital de género, por tal motivo la ONU considera, que la brecha digital de género femenino en el uso de Internet es del 12\% (ONU, 2019)

\section{Se necesitan más mujeres en la tecnología}

Elspeth McOmish, señala que, la única solución para resolver estos enigmas de la mujer en el diseño de la IA es que ellas formen parte fundamental en la elaboración de estos desafíos, y aunque también nos detalla que, "Efectivamente hay un problema desigualdad de género en la sociedad: ya sea por los contenidos impartidos en la educación y la pedagogía que son que hacen que muchas chicas no se sientan en confianza para hacer estudios de ciencias y matemáticas, y también en las familias y en muchos casos la desvalorización de las mujeres y de nuestras experiencias conduce a la banalización de la violencia de género", por tales motivos, la Unesco hace un llamado a todas las ciudadanas y ciudadanos a hacer presión sobre las empresas para que promuevan la igualdad de género a través de sus productos, de sus publicidades y las condiciones de trabajo con el único fin de que la presencia femenina puede influir mucho sobre la manera en que las tecnologías están creadas y qué el tipo de pensamiento varié de acuerdo al diseño de las aplicaciones de inteligencia artificial. (ONU, 2019)

\section{Razones por las que desaparecieron las mujeres en la ia y el mundo de la robótica}

Según Thompson, existen tres razones principales por las cuales hoy en día la mujer no está presente en la programación:

$\checkmark$ En primer lugar, el desarrollo del software era cada vez era más avanzado por ende las empresas buscaban a profesionales en ese ámbito para nombrarlos directivos, siendo elegidos en su gran mayoría hombres, dado que, si era mujer no tenía esa oportunidad de ser nombrada directiva.

$\checkmark$ En segundo lugar, la llegada del portátil a los hogares, puesto que, para los papás desde la niñez optan por ofrecerle este aparato a un hijo en vez de una hija, dado que, suponen que esté se desempeñaría mejor al seguir una carrera de informática, y es así como empieza la desigualdad de género.

$\checkmark \mathrm{Y}$, en tercer lugar, la llegada del dinero, cuando un sector es bien pagado. Los hombres siempre están un paso delante, puesto que, las mujeres diferencia de los hombres muchas veces se conforman al recibir un salario que no es el idóneo para sus cargos, mientras que ellos muestran mayor interés y son capaces de negociar o realizar pequeñas estrategias tecnológicas que les permite de alguna u otra forma mejorar el capital que se les ofrece. (PERÉZCOLOMÉ, 2019)

\section{Mujeres que han contribuido en la IA y robótica}

70 UNESUM-Ciencias. Publicación cuatrimestral. Vol. 5, Año 2021, No. 4 (Número Especial) 
Ada Lovelace, Karen Spärck Jones, Margaret Hamilton, Katherine Johnson, Mary Jackson y Dorothy Vaughan.

\section{La IA y la robótica impulsan la equidad de género por 5 razones}

La robótica se ha catequizado en una de las plazas de discernimiento más precoces para el progreso de los adolescentes. El campo de aprendizaje es muy amplio, lo cual permite que se adquiera no solo conocimientos, sino habilidades sobre la ciencia, la tecnología, la ingeniería y las matemáticas conocidos también como las conductas STEM. Por el contrario, la participación de las féminas en los campos de STEM es muy limitada, puesto que, existe un nivel bajo de estudiantes que optan por una licenciatura en vez de una ingeniería especializada en la tecnología. Ahora detallaremos las cinco razones que impulsaría la participación de las mujeres en las áreas informáticas:

1. Transforma el panorama sobre el desempeño de las mujeres en la ciencia

2. Motiva la colaboración entre hombres y mujeres

3. Empodera a las mujeres en habilidades STEM

4. Incrementa el desarrollo de habilidades de emprendimiento entre las mujeres

5. Inspira a más jóvenes

De esta forma, se suman esfuerzos para alcanzar los objetivos de la Agenda de Desarrollo Sostenible para 2030, en la que el promover la inclusión de las mujeres a partir de las habilidades STEM es una prioridad, con la finalidad de mejorar la vida de la población a nivel mundial con participación equitativa, innovación e impulso al crecimiento económico. (EL FINANCIERO, 2018)

\section{Iniciativas para desarrolladoras}

La comunidad R-Ladies, a nivel nacional, Girls in Tech, Women Techmakers, Geek and Tech Girls; y en Madrid, PyLadies, Women Welcome, Yo soy tu madre, Systers Spain, son algunas organizaciones que han dado inició a grandes proyectos con el único objetivo de aprender, hacer networking y promover a las mujeres programadoras. (Gonzalo, 2018)

Otra iniciativa con una finalidad similar es Ladies That UX, un evento mensual organizado en más de treinta ciudades del mundo que promueve la visibilidad de las mujeres en el área de la experiencia de usuario mediante la divulgación de sus trabajos y la creación de espacios de networking. Por consiguiente, tenemos a Inspiring Girls, una iniciativa dirigida por mujeres profesionales que inspiran a las niñas a aumentar su autoestima y ambición profesional. A través de visitas y charlas en las escuelas, les muestran a las niñas que ser mujer no es una limitación para desempeñar cualquier trabajo en el mundo; lo que sí importa es el esfuerzo dedicado a ello. (Codenotch, s.f.)

\section{Países donde el desempeño de la mujer es sumamente escaso}

En Argentina, el 90\% de los estudiantes de computación son varones, número que se replica, con mucha semejanza, a nivel global. Incluso en EE. UU el porcentaje de mujeres en trabajos vinculados a la tecnología y a las ciencias duras descendió en los últimos 23 años, según un 
estudio publicado por Forbes. En España los porcentajes entre estudiantes de informática son parecidos. En 1985, un 30\% de los alumnos de informática en España eran mujeres. En 2016 cayeron hasta el $12 \%$. Hoy, sólo el $25 \%$ de la fuerza laboral mundial del sector está representado por mujeres (Balbi, 2016).

\section{MATERIALES Y MÉTODOS}

Los materiales que llevaron a cabo en este trabajo de investigación fueron: diversas fuentes bibliográficas lo que conlleva a una investigación completa. En cuanto al desarrollo de esta indagación se utilizaron los métodos teóricos de la investigación científica tales como: históricológico que fue empleado en la construcción de todo el trabajo investigativo, el análisis-síntesis para profundizar y sintetizar el proceso de relación que se establece entre las mujeres, en el desarrollo de la inteligencia artificial en contribución con la robótica. Posterior a ello, se emplearon los métodos bibliográficos y referenciales para revisar antecedentes reales sobre el trabajo de investigación.

\section{DISCUSIÓN DE LOS RESULTADOS}

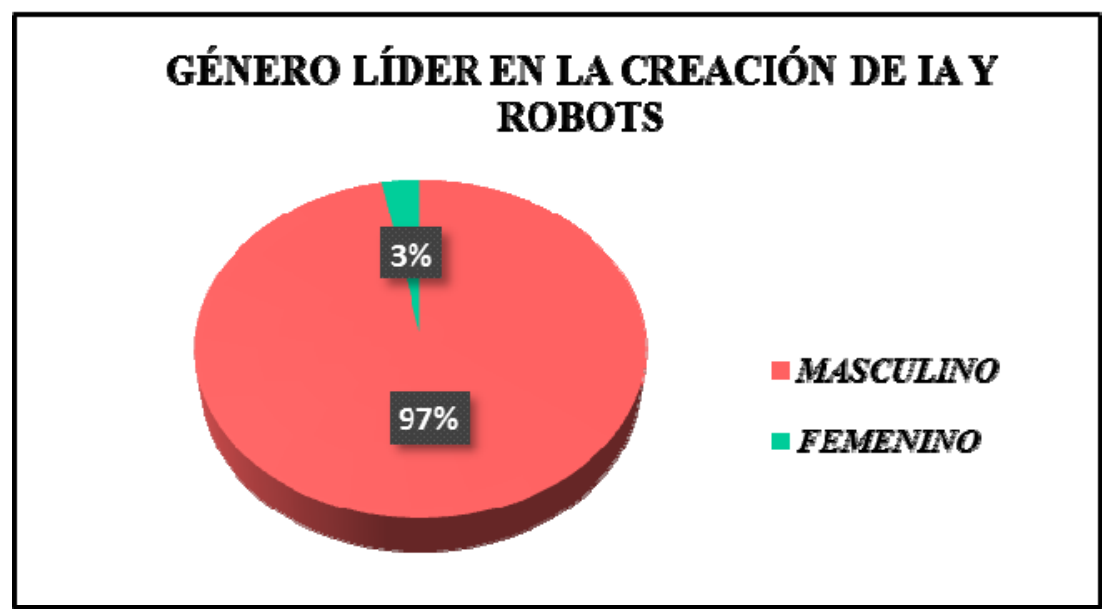

Figura 1: Datos del género líder en la creación de IA y Robots

\footnotetext{
Fuente: UNESCO

Elaboración: Reina Paredes John Denys, Pinargote Vinces Gema Julissa, Pin Menéndez Camila Yanlisbeth, Pérez Chilán Dayana Lilibeth, Marcillo Merino Mario Javier.
}

Una vez obtenidos los datos de la UNESCO, se constató que el 97\% del género masculino son líder en la creación de IA y Robots, mientras que tan solo el 3\% del género femenino son parte de aquellas creaciones.

\section{CONCLUSIONES}

Aunque la robótica es un campo mayoritariamente masculino, el desempeño de la mujer en estos procesos informáticos juega un papel individualizado dejando de lado que la mujer en el mundo

72 UNESUM-Ciencias. Publicación cuatrimestral. Vol. 5, Año 2021, No. 4 (Número Especial) 
Publicación cuatrimestral. Vol. 5, No. 4 (Número Especial), Año 2021. Pág. 67-74 DESEMPEÑO DE LA MUJER EN LA IA Y SU CONSTRIBUCIÓN CON LA ROBÓTICA

de la tecnología y la informática una de ellas fue considerada como la primera programadora de la historia.

Los avances en robótica prometen un futuro en el que se democratice y personalice a gran escala el acceso a máquinas, pero no suficiente para compensar la desigualdad de género que se puede encontrar en el ámbito tecnológico esta desigualdad queda patente, debido a que no se está siendo inclusivo con las mujeres en la tecnología.

En definitiva, ni la genética, ni la falta de interés, es la causa de que exista un bajo desempeño de la mujer en el desarrollo de la IA en contribución con la robótica, sino del contenido educativo y pedagógico que reciben los adolescentes desde su infancia, dado que, no existe una igualdad de género donde la mujer sea capaz de promover proyectos relacionados directamente con la ciencia, tecnología, ingeniería y matemáticas.

\section{REFERENCIAS BIBLIOGRÁFICAS}

Balbi, M. (08 de marzo de 2016). infobae.com. Obtenido de Las mujeres y la tecnología: https://www.infobae.com/2016/03/08/1795540-las-mujeres-y-la-tecnologia/

Codenotch. (s.f.). codenotch.com. Obtenido de Codenotch: https://codenotch.com/blog/donde-estan-las-mujeresprogramadoras/

EL FINANCIERO. (16 de abril de 2018). elfinanciero.com.mx. Obtenido de La robótica impulsa la equidad de género por estas 5 razones: https://www.elfinanciero.com.mx/universidades/la-robotica-impulsa-la-equidadde-genero-por-estas-5-razones

Gonzalo, M. (14 de junio de 2018). elpais.com. Obtenido de Mujeres programadoras contra los estereotipos: adivina quién va ganando: https://elpais.com/tecnologia/2018/06/14/actualidad/1528977394_341784.html

ONU. (3 de junio de 2019). news.un.org. Obtenido de La ausencia de mujeres en el campo de la inteligencia artificial reproduce el sexismo: https://news.un.org/es/story/2019/06/1456961

Peiró, K. (09 de noviembre de 2019). karmapeiro.com. Obtenido de ¿Por qué la inteligencia artificial discrimina a las mujeres?: $\quad$ https://www.karmapeiro.com/2019/11/09/por-que-la-inteligencia-artificial-discrimina-a-lasmujeres/

PERÉZCOLOMÉ, J. (11 de septiembre de 2019). elpais.com. Obtenido de Cuando las mujeres dominaban el software: https://elpais.com/tecnologia/2019/09/11/actualidad/1568211819_635943.html 